\title{
Focusing of Terahertz Waves onto Micron-sized Slits Grown on ZnTe and GaP Substrates
}

\author{
M. A. Seo, H. R. PArk, D. J. PArk and DaiSik KIM* \\ Center for Subwavelength Optics and Department of Physics and Astronomy, Seoul National University, Seoul 151-747 \\ A. J. L. Adam and P. C. M. Planken \\ Faculty of Applied Sciences, Delft University of Technology, Lorentzweg 1, 2628 CJ Delft, The Netherlands
}

(Received 26 August 2008)

\begin{abstract}
We report on terahertz focusing through rectangular slit apertures on thin metal film by using terahertz time domain spectroscopy both in the far- and in the near-field. Our experiments show that far-field perfect transmission is maintained in the rectangular slit array even as the aperture coverage decreases. Near-field imaging supports the field enhancement in the single rectangular aperture indeed occurring at a frequency equal to the fundamental shape resonance frequency.
\end{abstract}

PACS numbers: 84.40.Az, 07.57.Kp, 32.30.-r

Keywords: Terahertz, Optical spectroscopy, Plasmonic metamaterials

\section{INTRODUCTION}

Plasmonics has attracted growing attention in the photonics community in the last decade [1]. The concept of the surface plasmon polariton has been extended to perforated structures with local resonances in the microwave and the terahertz frequency ranges where metals are highly conducting [2-5]. In perfect metals, the lightning rod effect becomes singular and a large field enhancement is expected. Recently, it was predicted [6] that a rectangular aperture with a large aspect ratio at its fundamental resonance would focus the incoming wave onto its small area through an enormous field enhancement. Here, we performed terahertz far-field [7] and near-field electro-optic sampling experiments [8] on free-standing rectangular hole arrays and on a single rectangular aperture grown on an electro-optic crystal substrate, GaP, respectively. We found that far-field transmission achieved perfect transmission at the fundamental shape resonance, regardless of the area coverage of the holes, as long as the aspect ratio of the rectangle was sufficiently large. Indeed, the near-field imaging demonstrated that the electric field enhancement really existed. We expect that the enormous field enhancement will have wide-ranging implications in many areas where strong terahertz fields are much desired. This type of THz field energy focusing is implied in many applications, including perfect transmission in perfect conductors with apertures [9], near-field lithography using nano-wires [10], plasmonic nanolithography [11], super lensing [12,13],

*E-mail: dsk@phya.snu.ac.kr; Fax: +82-2-889-1295 and a recently proposed evanescent-field lens [14].

\section{EXPERIMENTS AND DISCUSSION}

It is notable that the earlier theoretical study by García-Vidal et al. [6] on rectangle apertures assumed a perfectly conducting metal, which is readily applicable to the $\mathrm{THz}$ or the microwave region. Recently, the shape resonance was shown to be the dominant effect responsible for the extraordinary transmission through metal sheets with subwavelength rectangle holes in the $\mathrm{THz}$ spectral region [15]. While the far-field experiment showed terahertz transparency at the fundamental shape resonance of the rectangular holes in the far-field region, the near-field measurement directly and quantitatively displays strong enhancement around a single rectangle.

Consider a rectangular aperture whose width is much smaller than the incoming light's wavelength in a thin, perfectly conducting film with the incident polarization perpendicular to the length direction. Different from a circular hole, which has been addressed and exactly solved in many diffraction theories ever since Bethe [16] and Bouwkamp [17], the rectangle shape exhibits many shape resonances $[15,18,19]$.

We use a THz TDS (time-domain spectroscopy) system to measure the far-field spectrum and utilize a recently developed Fourier-transform terahertz near-field microscopic technique [20] to probe the time and the frequency domain near-field images, respectively. Both systems are based on the EO (electro-optic) sampling technique to detect a $\mathrm{THz}$ signal with a working frequency 
(a) THz far-field spectroscopy
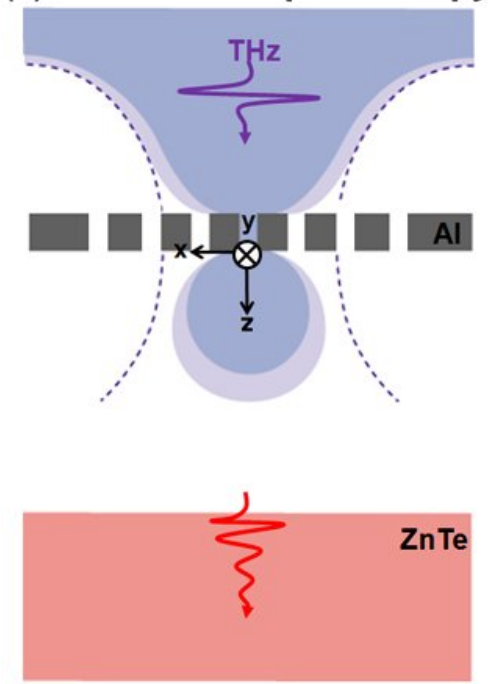

\section{(b) THz near-field imaging}

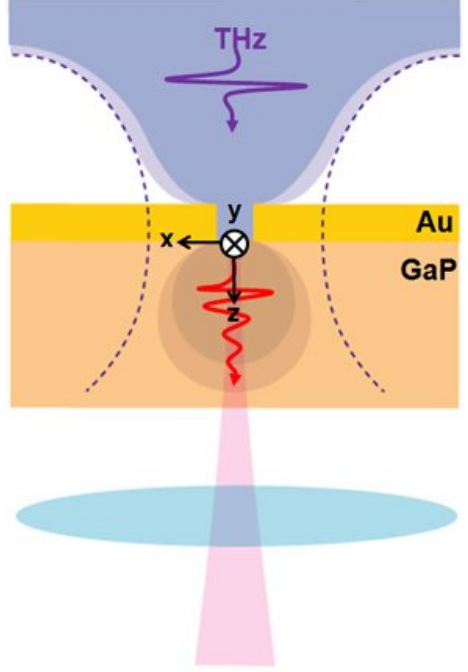

Fig. 1. (a) Schematic of the $\mathrm{THz}$ far-field spectroscopy. The $\mathrm{THz}$ wave transmitted through free standing metal slits is measured by using electro-optic sampling with a (110) ZnTe crystal. (b) Terahertz near-field measurement of a single rectangular hole of sides $a_{x}$ and $a_{y}$ perforated on a gold metal film deposited on a piece of GaP electro-optic crystal. The crystal with the metal sample was raster scanned with respect to the probe/ $\mathrm{THz}$ beam to image the near surface of the transmitted side. Both systems were normally irradiated by using an $x$-polarized terahertz plane wave, and electro-optic sampling was performed using terahertz time domain spectroscopy.

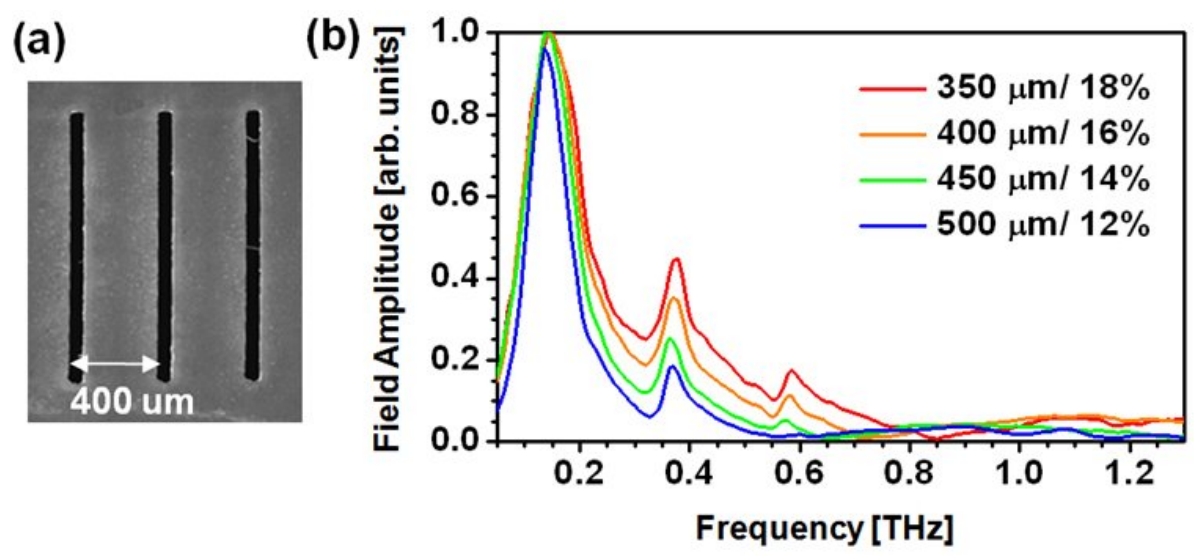

Fig. 2. (a) SEM image of rectangle array with a length of $1250 \mathrm{um}$ and a period of $400 \mathrm{um}$. (b) Normalized transmission, at normal incidence, of rectangular holes with the same length of $1250 \mathrm{um}$, but with different periods of 350, 400, 450, and 500 um corresponding, respectively, to areal coverages of $18 \%, 16 \%, 14 \%$, and $12 \%$.

between $0.05-1.3 \mathrm{THz}$. Using two differently-oriented GaP crystals, we were able to independently measure all three vector components of the terahertz electric field [21]. Especially, the electro-optic sampling technique with a (110) orientation allowed us to detect only the component of the $\mathrm{THz}$ electric field parallel to the crystal surface $[22,23]$. To examine the focusing effect at the hole precisely, we measured only the component of the electric field parallel to the crystal surface while the incident $\mathrm{THz}$ light was polarized along the $x$-axis (Figs. $1(\mathrm{a})$ and $1(\mathrm{~b})$ ). Particularly, in near-field imaging systems (Fig. 1(b)), while the $\mathrm{THz}$ wave impinges on the structured metal, the probe pulse sees the interface be- tween the deposited metal film and the detection crystal and the probe beam size results in a spatial resolution of less than $10 \mathrm{um}$. Because the metal surface is in direct contact with the detection crystal, this measurement addresses only the near-field near the surface of the metal $(z=0)$.

As in an earlier prediction [4], for the polarization chosen, the transmittance peak appearing at the cutoff depends only on the long side $\left(\lambda_{\text {res }}=2 a_{y}\right)$, and the ratio between the long side and the short side of the rectangle gives rise to the area-normalized transmittance, $T_{\text {res }} \approx(3 / \pi) a_{y} / a_{x}$, close to the cutoff. Therefore, the question is as the coverage, meaning the distance be- 

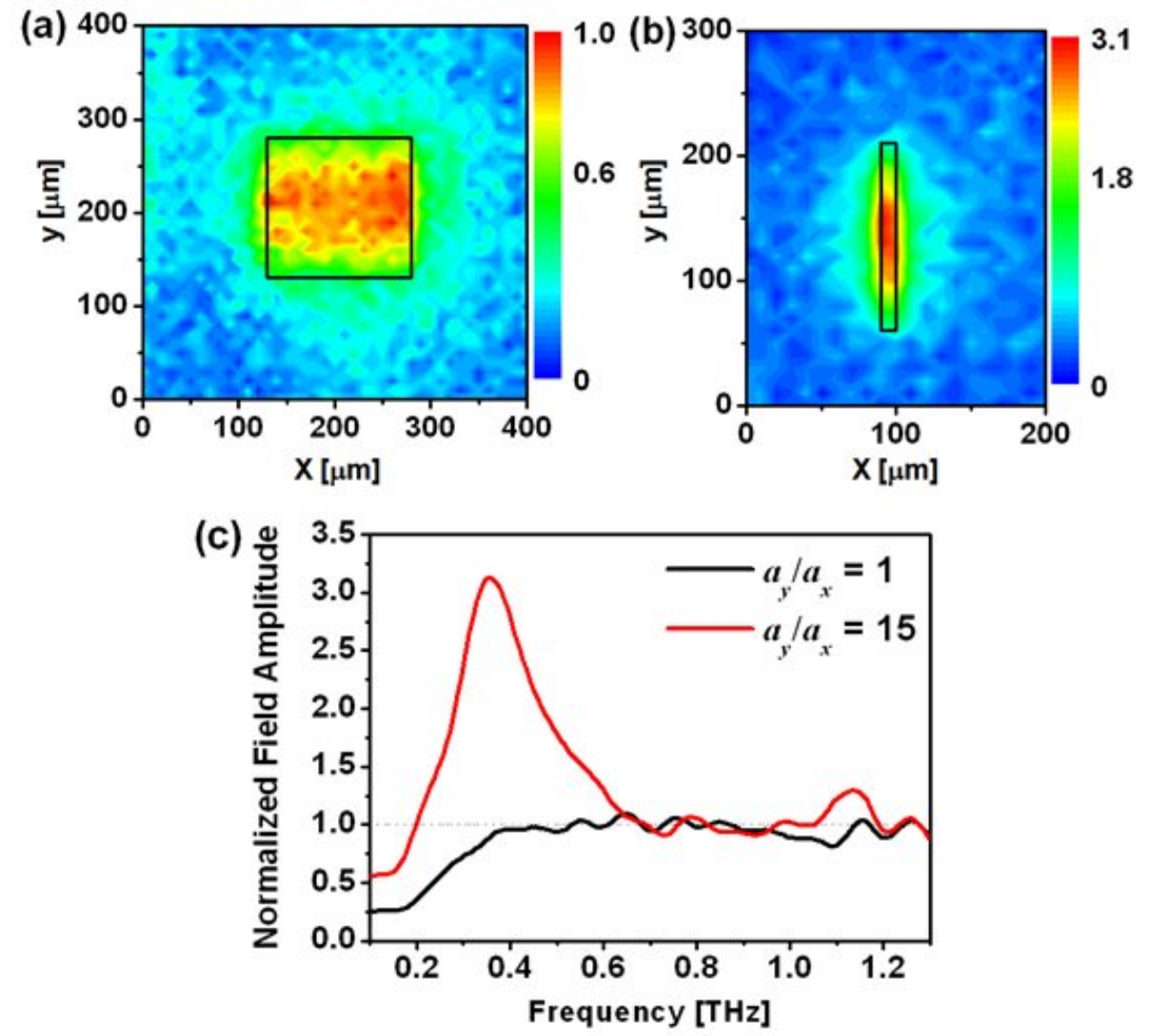

Fig. 3. (a) Fourier-transformed image of the $E_{x}$ component for a $150 \mathrm{um}$ by $150 \mathrm{um}$ square at $0.37 \mathrm{THz}$, (b) Fouriertransformed image of the $E_{x}$ component of a rectangle with a 10-um width and a 150-um length at $0.37 \mathrm{THz}$. (c) Field amplitude spectra for the square and the rectangle after area normalization.

tween adjacent slits, changes, perfect transmission be maintained? As shown in Fig. 2(b), near-perfect transmission is maintained at the fundamental shape resonance even as the distance between apertures becomes larger. The transmittance above the first Rayleigh minimum, $f_{R}=c / d=0.16 \mathrm{THz}$, in the frequency range of approximately 0.8 to $1.3 \mathrm{THz}$ is roughly equal to the aperture area coverage, where $d$ is a period of the slit array.

To precisely investigate the role of a rectangular shape in the field enhancement, we performed $\mathrm{THz}$ near-field imaging with a sample grown directly onto the detection crystal. We scan the $x$-component of the electric field around on aperture having a scan area of $400 \mathrm{um} \times 400$ um for the square aperture and of $200 \mathrm{um} \times 300 \mathrm{um}$ for the rectangle in the $x-y$ plane with a spatial step size of $20 \mathrm{um}$. In order to compare the effect of the rectangular ratio on the transmittance, we designed the rectangles with different short sides of $a_{x}=150 \mathrm{um}$ (Fig. 3(a)) and $10 \mathrm{um}$ (Fig. 3(b)) for a fixed long side $a_{y}=150 \mathrm{um}$, which results in a fundamental shape resonance peak at $0.37 \mathrm{THz}$. We plot the $x$-component of the electric field, $\overrightarrow{E_{x}}(x, 0, z, t)$ at $0.37 \mathrm{THz}$ to show that $E$-field focusing occurs around the aperture with a size of $10 \mathrm{um}$ along the $x$-axis and $150 \mathrm{um}$ along the $y$-axis $\left(a_{y} / a_{x}=15\right)$, as shown in Fig. 3(b). These near-field amplitudes are normalized against the higher frequency backgrounds, which remain virtually constant over the frequency range of 0.8 $-1.3 \mathrm{THz}$ for these two samples. This field image reveals the strongly localized character of the field at the center of the rectangular aperture. Clearly, as shown in this figure, a transmission peak develops at approximately $\lambda_{\text {res }}$ $=2 a_{y} / n$, with the aspect ratio, $a_{y} / a_{x}$, being much larger than 1 (15 for the case of Fig. 3(b)). It is notable that the peak amplitude of the electric field increases as the ratio increases, as predicted by the earlier theory [6].

\section{CONCLUSION}

In conclusion, we investigated perfect transmittance through rectangular aperture arrays at the fundamental resonance frequency as a function of the aperture coverage. A comparison between the near-field imaging for the square and the rectangular single aperture shows that a large aspect ratio between the length and the width of the rectangle can support stronger field focusing around the center of the structure. The areanormalized spectra show that at the resonance peak, the areanormalized 
transmittance and therefore the normalized amplitude are greater than 3 for a rectangle with an aspect ratio of 15. The extremely-large-aspect-ratio rectangular holes therefore, with their large field amplitude enhancements, will find immediate application in physics, engineering, and biology, where large and controllable terahertz field enhancement is much desired.

\section{ACKNOWLEDGMENTS}

This work was supported by the Korea Science and Engineering Foundation (KOSEF) grant funded by the Korea government (MEST), the Korea Research Foundation (KRF), KICOS (GRL, K20815000003) and the Seoul R\&BD Program.

\section{REFERENCES}

[1] T. W. Ebbesen, H. J. Lezec, H. F. Ghaemi, T. Thio and P. A. Wolff, Nature 391, 667 (1998).

[2] A. P. Hibbins, B. R. Evans and J. R. Sambles, Science 308, 670 (2005).

[3] J. B. Pendry, L. Martin-Moreno and F. J. Garcia-Vidal, Science 305, 847 (2004).

[4] J. H. Kang, Q.-H. Park, J. W. Lee, M. A. Seo and D. S. Kim, J. Korean Phys. Soc. 49, 881 (2006).

[5] J. W. Lee, M. A. Seo, J. Y. Sohn, D. J. Park, Y. H. Ahn, S. C. Jeoung, Q.-H. Park and D. S. Kim, J. Korean Phys. Soc. 48, 103 (2006).

[6] F. J. Garcia-Vidal, E. Moreno, J. A. Porto and L. Martin-Moreno, Phys. Rev. Lett. 95, 103901 (2005).
[7] M. A. Seo, H. R. Park, S. M. Koo, D. J. Park, J. H. Kang, O. K. Suwal, S. S. Choi, P. C. M. Planken, G. S. Park, N. K. Park, Q. H. Park and D. S. Kim, Nature Photonics 3, 152 (2009).

[8] M. A. Seo, A. J. L. Adam, J. H. Kang, J. W. Lee, K. J. Ahn, Q. H. Park, P. C. M. Planken and D. S. Kim, Opt. Express 16, 20484 (2008).

[9] J. W. Lee, M. A. Seo, J. Y. Sohn, Y. H. Ahn, D. S. Kim, S. C. Jeoung, Ch. Lienau and Q.-H. Park, Opt. Express 13, 10681 (2005).

[10] D. Li and Y. Xia, Nat. Mater. 3, 753 (2004).

[11] W. Srituravanich, N. Fang, C. Sun and X. Zhang, Nano. Lett. 4, 1085 (2004).

[12] N. Fang, H. Lee, C. Sun and X. Zhang, Science 308, 534 (2005)

[13] D. O. S. Melville and R. J. Blaikie, Opt. Express 13, 2127 (2005)

[14] R. Merlin, Science 317, 927 (2007).

[15] J. W. Lee, M. A. Seo, D. H. Kang, K. S. Khim, S. C. Jeoung and D. S. Kim, Phys. Rev. Lett. 99, 137401 (2007).

[16] H. A. Bethe, Phys. Rev. 66, 163 (1944).

[17] C. J. Bouwkamp, Philips Res. Rep. 5, 401 (1950).

[18] K. L. van der Molen, K. J. Koerkamp, S. Enoch, F. B. Segerink, N. F. Hulst and L. Kuipers, Phys. Rev. B 72, 045421 (2005).

[19] H. Cao and A. Nahata, Opt. Express 12, 3664 (2004).

[20] M. A. Seo, A. J. L. Adam, J. H. Kang, J. W. Lee, S. C. Jeoung, Q. H. Park, P. C. M. Planken and D. S. Kim, Opt. Express 15, 11781 (2007).

[21] P. C. M. Planken, H. K. Nienhuys, H. J. Bakker and T. Wenckebach, J Opt. Soc. Am B 18, 313 (2001).

[22] N. J. C. van der Valk, T. Wenckebach and P. C. M. Planken, J. Opt. Soc. Am. B 21, 622 (2004).

[23] D. F. Nelson and E. H. Turner, J. Appl. Phys. 39, 3337 (1968). 